\title{
Methodological Survey on Fetal ECG Extraction
}

\author{
Anisha.M ${ }^{1}$, Dr.S.S.Kumar ${ }^{2}$ Benisha.M ${ }^{3}$ \\ ${ }^{I}$ Department of BioMedicalEngineering,Noorul Islam University, kumaracoil, 629180,Tamil Nadu,India \\ ${ }^{2}$ Department of Electronics \&Instrumentation Engineering,Noorul Islam \\ University,kumaracoil,thuckalay,India. \\ ${ }^{3}$ Department of Electronics \& Communication Engineering, Jeppiaar Institute of Technology, Chennai
}

\begin{abstract}
Fetal Electrocardiogram (FECG) signal, non-invasively taken from the Abdominal Electrocardiogram (AECG) of a pregnant woman is a efficient diagnostic tool for evaluating the health status of fetus. Clinically significant information in the Fetal Electrocardiogram signal is often masked by Maternal Electrocardiogram (MECG) considered as the most predominant interference, power line interference, and maternal Electromyogram (EMG), baseline wander etc. Fetal Electrocardiogram signal features may not be readily comprehensible by the visual or auditory systems of a human. Therefore Fetal Electrocardiogram should be extracted from composite Abdominal Electrocardiogram for clinical diagnosis. There are many powerful and well advanced methods for this purpose. A methodological study has been carried out to show the effectiveness of various methods which helps in understanding of Fetal ECG signal and its analysis procedures by providing valuable information.
\end{abstract}

Keywords: AbdominalECG, MaternalECG, ElectroMyoGram

\section{Introduction}

Heart defects are being the most common birth defects and the leading cause of sudden prenatal death. [1],[2].The cardiac defect may be very slight so that the baby appears healthy and normal for many years after birth, but suddenly becomes so severe due to that its life is in immediate danger. Congenital heart defects originate when the heart is forming and they can affect any of the parts or functions of the heart. Cardiac anomalies may occur due to a inherited disorder, genetic syndrome, or environmental factors such as infections or misuse of drug during pregnancy [4],[1]. Every year one out of 125 babies are born with some kind of congenital heart defects [3]. FECG carries vital information about the cardiac function of fetus. The characteristics of the fetal electrocardiogram (FECG),such as heart rate, waveform, and dynamic behavior, are important in determining the fetal life, fetal development, fetal maturity, and existence of fetal distress or congenital heart disease.Fetal electrocardiography has proved an effective tool for imaging specific structural defects only at the time of labor not during pregnancy because FECG [5] is contaminated by fetal brain activity,myographic (muscle) signals (from both the mother and fetus), movement artifacts and multiple layers of different dielectric biological media through which the electrical signals must pass. Fetal monitoring is based entirely on the fetal heart rate and does not incorporate characteristics of the fetal ECG (fECG) waveform that are the cornerstone of cardiac evaluation. The main reason for this is there is no available technology to reliably measure fECG. Most of the heart defects have some manifestation in their morphology, which is believed to contain much more information as compared with other conventional methods [6]. Most of the clinically useful information in the FECG signal is found in the amplitude and duration of its waveforms [7]. Fetal cardiac waveform helps physicians to diagnose fetal heart arrhythmia such as Bradycardia, Tachycardia, Asphyxia and Hypoxia. It has long been recognized that persistent of fetal tachyarrhythmia and bradyarrhythmia may also lead to the evolution of heart failure [8-12] and may be associated with neurological injury [13],[14].

\section{Methods}

There are many methods for extracting FECG from AECG such as Adaptive filtering[15]-[20], wavelet Transform[30]-[41], soft computing tools[42]-[57] like Adaptive neural network, Genetic Algorithm, Adaptive Neuro Fuzzy Inference System, Support Vector Machine[58]-[74], Independent component Analysis [75]-[85], etc.

\subsection{Adaptive Filtering}

An adaptive filter is a filter that self-adjusts its transfer function according to an optimization algorithm driven by an error signal.

The composite input signal is represented as

$$
\mathrm{X}(\mathrm{n})=\mathrm{v}(\mathrm{n})+\mathrm{m}(\mathrm{n})
$$


Where

$\mathrm{v}(\mathrm{n})$ is signal of interest.

$\mathrm{m}(\mathrm{n})$ is major mater interference overlapped with the signal of interest.

Adaptive noise canceller shown in Fig [1] requires a secondary input also known as reference input $r(n)$ that should be uncorrelated with signal of interest and closely correlated with the interference. Adaptive filter learns and adapts the characteristics of reference signal and modifies it exactly similar to the influencing interference $y(n)$.

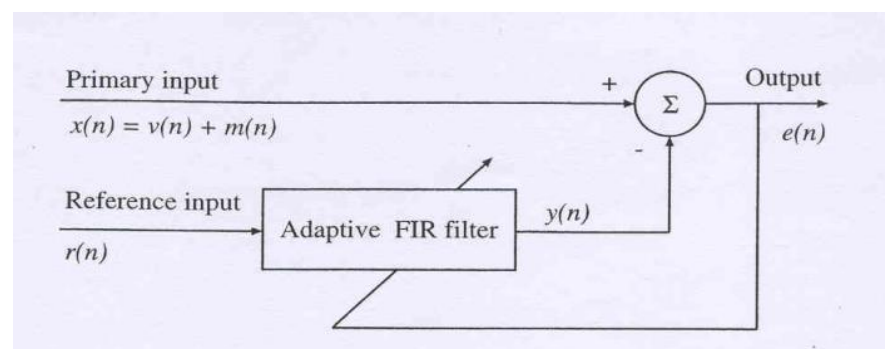

Fig:1 Adaptive noise canceller

Assume that $v(n), m(n), r(n), y(n)$ are stationary and have zero means.

$$
\begin{aligned}
& e(n)=x(n)-y(n) \\
& =v(n)+m(n)-y(n)
\end{aligned}
$$

$\mathrm{y}(\mathrm{n})=\sim \mathrm{m}(\mathrm{n})$ is the estimate of the primary noise obtained at the output of the adaptive filter.

Taking the square and expectation(statistical average)

$$
\left.\mathrm{E}\left[\mathrm{e}^{2}(\mathrm{n})\right]=\mathrm{E}\left[\mathrm{v}^{2}(\mathrm{n})\right]+\mathrm{E}\left[\{\mathrm{m}(\mathrm{n})-\mathrm{y}(\mathrm{n})\}^{2}\right]+2 \mathrm{E}[\mathrm{v}(\mathrm{n})\{\mathrm{m}(\mathrm{n})-\mathrm{y}(\mathrm{n})\}]\right)
$$

Since $m(n)$ and $y(n)$ are uncorrelated with $v(n)$

$$
\begin{aligned}
& E[v(n)\{m(n)-y(n)\}]=E[v(n)] E[m(n)-y(n)]=0 \\
& E\left[e^{2}(n)\right]=E\left[v^{2}(n)\right]+E\left[\{m(n)-y(n)\}^{2}\right] \\
& \min E\left[e^{2}(n)\right]=E\left[v^{2}(n)\right]+\min E\left[\{m(n)-y(n)\}^{2}\right]
\end{aligned}
$$

When $E\left[e^{2}(n)\right]$ is minimized, min $E\left[\{m(n)-y(n)\}^{2}\right]$ is also minimized When $E\left[\{e(n)-v(n)\}^{2}\right]=0$ Output SNR is improved.

Minimizing the total output power minimizes the output noise power and maximizes the output SNR.

Different types of adaptive filters have been used for fetal and maternal signal separation. These methods use one or more reference maternal signals[15]-[29] for training an adaptive or matched filter, or directly training the filter without reference signal [19],[20] for extracting the fetal QRS waves. Ad hoc filters such as least square error fittings [21] and partition-based weighted sum filters [22] have also been used for FECG extraction. The kalman filter, a general class of adaptive filter used in [23]-[26] uses only an arbitrary MECG as reference for MECG cancellation and FECG extraction.

In [23] and [27], a set of state-space equations was used to model the temporal dynamics of ECG signals, for designing a Bayesian filter for ECG denoising. This Bayesian filter framework was used in [23] to extract fECG from single channel mixture of mECG and fECG. However, as mentioned in [23], the filter fails to discriminate between the maternal and fetal components when the mECG and fECG waves fully overlap in time. The reason is that when mECG is being estimated, fECG and other components are supposed to be Gaussian noises.However, this assumption is not true, especially when mECG and fECG waves fully overlap in time it is difficult for the filter to follow desired ECG.

An improved method proposed in [28] uses multistage adaptive filtering for FECG extraction in which MECG cancellation has been done by considering thoracic ECG as reference signal also denoising methods were used to improve the quality of extracted signal.A linear adaptive filter [29] was used to extract the FECG by considering abdominal ECG as primary inputs where as thoracic ECG taken from maternal chest as reference 
inputs. Though this method provides a solution it fails to extract when maternal and fetal signals are overlapped each other. So it is not best for clinical practice.

\subsection{Wavelet Transform}

The Wavelet Transform is a time-scale representation also best tool for analyzing non-stationary signals. Wavelet transform of a signal is defined as

$$
W_{x}(s, t)=x(t) o \psi_{s}(t)=\frac{1}{s} \int_{-\infty}^{+\infty} x(\tau) \cdot \psi\left(\frac{t-\tau}{s}\right) d \tau
$$

where

(o) = convolution operation,

$\psi(t)=$ basis function (called mother wavelet)

$\mathbf{s}=$ scale factor.

$\mathbf{x}(\mathbf{t})=$ input signal

Wavelet technique was applied in [30] to detect the presence of distorted MECG signal and then MECG component has eliminated from the composite signal. Sometimes even after the elimination of MECG, FECG observation was still complicated and challenging because the wavelet analysis could enhance FECG signal alone. It also detects the singularity of signal either in time or frequency domain. The method developed in [31] uses wavelet analysis and pattern matching, in which at preprocessing stage low and high frequency noises in the AECG signal was suppressed based on optimal wavelet multi resolution decomposition and then maternal QRS complexes were cancelled by means of pattern matching and template subtraction. Then by applying a QRS detection algorithm, fetal QRS complexes were identified.

FECG was separated and monitored in [32] by calculating lipschitz exponent. This method fails to locate the FECG, if it was obscured by the MECG, which continues more than 2 times in a 10-s period that may be a main drawback of this method. Also there is a need to set the thresholds on the wavelet co-efficients dynamically due to the existence of more noise content because of uterine contraction. Wavelet transform based approach proposed in [33]efficiently eliminates transient spikes and reduces both Gaussian and colored noise without affecting or destroying the information content of the signal. Noise components were detected by analyzing the evolution of the WT modulus maxima across scales. Multi-scale maxima that correspond to noise components were eliminated then de-noised fetal signal was reconstructed by taking inverse WT.Wavelet based multi resolution analysis(MRA) has developed in [34]to remove the base line wander and the other existing noises, Daubechies 20 wavelet function with 12 levels of decomposition was performed on the raw ECG data. Noise conponents were eliminated by thresholding the wavelet co-efficient at each level.

Complex continuous wavelet transform (CCWT) based technique has implemented in [35] along with modulus maxima theory to detect fetal QRS complexes from multi channel MECG recordings. CCWT was used to identify stationary sections and to locate as well as characterize singularities.In [36] similar type of technique was proposed where fetal phono cardio signals were detected and denoised. This method improved the signal to noise ratio, and estimated FHR variation under very weak signal environment. In [37],FHR extraction from composite AECG signal has been done based on time frequency analysis, procedure composed of three stages. In the first stage maternal QRS complexes were eliminated by detecting maternal R-peaks and fiducial points using time-frequency analysis. At the second stage fetal $\mathrm{R}$ peaks were located using complex wavelets and pattern matching techniques, finally based on histogram technique overlapped fetal $\mathrm{R}$ peaks were detected at the third stage.Combination of Wavelet and ICA,called WICA technique proposed in [38]used each row data into an n-dimensional orthogonal basis. The extended-INFOMAX is a learning rule which was used to compute the demixing matrix. By this algorithm the independent components (ICs) were extracted and the ICs accounting for FECG was selected. Using WICA method FHR was detected and the Q, R, and S waves were visible without any signal amplification.

An algorithm proposed in [39] to design a new mother wavelet (MW) called abdominal ECG mother wavelet(AECG MW) for extraction of fetal ECG. Unlike other MWs which were used in extraction of fetal ECG, this newly proposed MW was designed to have a shape similar to AECG. To design such an MW, at least eight Gaussians should be used to model the 4 peaks in QRST of MECG and 4 peaks in QRST of FECG.A way for detecting QRS complex based on dyadic wavelet transform (D WT y ) has represented in[40]. They have designed a Spline wavelet for detecting QRS complex which was the transient part in the ECG signal.Here detection process was based on "the property that the absolute value of D WT y has localized maxima across several consecutive scales at the instant of the occurrence of transient." In this case, for each scale they found the local maxima of the absolute value of dyadic wavelet transform by using threshold method,the position of 
each local maxima was considered to be the location of a QRS complex. They have computed the heart rate by calculating the inverse of the time interval between two consecutive $\mathrm{R}$ waves.Real time fetal electrocardiogram (FECG) feature extraction system was developed in [41]based on multi-scale discrete wavelet transform (DWT). Wavelet based peak detection detects QRS complex more accurately for identifying peaks and valleys of noisy FECG signal. Two channel perfect reconstruction (PR) filter banks were used to implement the efficient way discrete wavelet transform.

\subsection{Artificial Intelligence Techniques}

ANFIS is a soft computing tool to estimate the non-linear transformations. To represents the ANFIS architecture, two-fuzzyRules are considered based on a first-order Sugeno model,

Rule 1: if ( $\mathrm{x}$ is $\mathrm{A} 1)$ and ( $\mathrm{y}$ is $\mathrm{B} 1)$,

then $(\mathrm{f} 2=\mathrm{p} 1 \mathrm{x}+\mathrm{q} 1 \mathrm{y}+\mathrm{r} 1)$

Rule 2: if ( $\mathrm{x}$ is $\mathrm{A} 2$ ) and (y is $\mathrm{B} 2)$,

then $(\mathrm{f} 2=\mathrm{p} 2 \mathrm{x}+\mathrm{q} 2 \mathrm{y}+\mathrm{r} 2)$

Layer 4

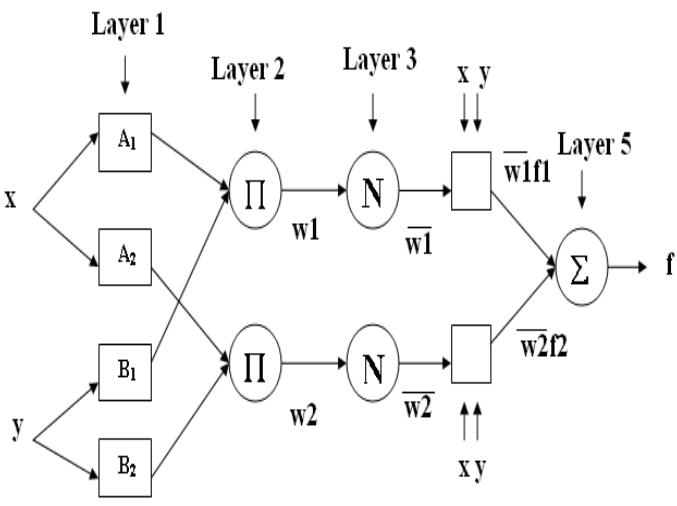

Fig:2 Adaptive neuro Fuzzy inference system

One possible ANFIS architecture to implement these two rules is shown in Fig. 2.in which fixed node is represented by circle whereas an adaptive node is indicated by square (the parameters can be changed during the process of training).Each layer has its own computation.

Layer 1: Calculate Membership Value for Premise Parameter

Layer 2: Firing Strength of Rule

Layer 3: Normalize Firing Strength

Layer 4: Consequent Parameters

Layer 5: Overall Output

The ANFIS architecture is not unique. Some layers can be combined to produce the same output.

In [42], back propagation network and the SOM network were used to categorize the FHR patterns.A new method for FHR baseline determination using ANNS was proposed in [43]. Two methods namely baseline estimation and baseline classification with multi layer perception artificial neural networks were applied on AECG data. Results obtained by these approaches were compared based on their practical application. In [44] a method was implemented with neural and fuzzy classifiers to differentiate the normal and pathological fetal states also to improve the diagnostic information contained in CTG signals.In [45] neural network real time recurrent learning algorithm was applied for training which converges faster to a lower mean squared error and also it is well suitable for real-time processing.In [46] FIR neural network was used for FECG extraction, in order to provide highly nonlinear dynamic capabilities. In this method thoracic maternal signal was considered as a reference signal, doesn't have fetal contributions and the desired signal was composite abdominal ECG signal. Though in [47] many reference signals had taken into consideration, the proposed method has considered only one thoracic signal.

In [48] adaptive linear neural network based FECG extraction method was proposed which trained the input composite AECG signal to vanish out the maternal signal therefore fetal ECG signal was isolated. Fetal heart is so small when compared with the maternal heart so the electrical activity produced by fetal heart is 
much weaker than the maternal due to its domination nature, MECG can be estimated easily then subtracted from the AECG, to get FECG. When compared to the conventional filtering techniques this produce better result because instead of elimination subtraction was used. In [49] signal processing tools and neural networks were used to develop an automated technique to detect the FHR pattern of baseline, acceleration and deceleration. However, ANN based FECG extraction methods have advantages also drawbacks, such as non-convex quadric minimization, which may result in multiple minima and the risk of over fitting [50].

A new method described in [51] using ADALINE for FECG extraction which emulates maternal signal as closely as possible to abdominal signal, thus only predict the maternal ECG in the abdominal ECG. The network error equals abdominal ECG minus maternal ECG, which is the fetal ECG. The characteristic that enables fetal extraction is due to the correlation between maternal ECG signals and the abdominal ECG signal of pregnant woman.A method described in [52] combines ANFIS and genetic Algorithm to extract FECG, Here ANFIS has been used to determine the non linear transformation (MECG).FECG is extracted by Subtracting the aligned version of the determined MECG signal from the abdominal ECG (AECG) signal.

In [53] an adaptive method was described to extract FECG by using adaptive linear neural network (ADALINE) for easy fetal monitoring in which learning rate, momentum and initial weights were adjusted until the network error equals fetal ECG.This method performed well with high learning rate and low momentum and small initial weights. The method proposed in [54] uses Adaptive Nero-Fuzzy Inference System (ANFIS) structure to identify the nonlinear transformation,here the maternal component of the abdominal ECG is a nonlinearly transformed version of MECG.Particle Swarm Optimization (PSO)also considered as a new tool for training the ANFIS structure. By identifying the nonlinear transformation, FECG was extracted by subtracting the aligned version of the MECG signal from the abdominal ECG (AECG) signal.

In [55] Genetic Algorithm (GA) acts as a tool for training the ANFIS structure, which identifies the non-linear transformation. By identifying the nonlinear transformation, FECG extraction is done by subtracting the aligned version of the MECG signal from the abdominal ECG (AECG) signal. In[56] adaptive neuro-fuzzy inference system (ANFIS) for Fetal Electrocardiogram extraction was presented.ANFIS is used to nonlinearly align the maternal ECG signal with the components of maternal ECG in the abdominal ECG signal. Hence identified maternal components were cancelled from the abdominal ECG signal and finally Fetal ECG signal was extracted.

In [57], an adaptive neuro-fuzzy logic method has proposed for FECG extraction and MECG cancellation. Rather than using conventional filtering techniques, adaptive filter was used for noise cancellation in the proposed technique, because it is a self adjusting filter, can change according to the environment and also when the signal was passed through a filter it suppress the nose and the signal remains unchanged while leaving the filter. Also this filter does not require a prior knowledge of signal or noise characteristics.

\subsection{SVD, ICA And Blind Source Seperation}

Recently, Support Vector Machines (SVM) developed by Vapnik, have gained popularity due to many attractive features capable of overcoming the limitations compared to the ANN. This is due to the Structural Risk Minimization (SRM) principle embodied by the SVM, which has been demonstrated to be more effective than the traditional Empirical Risk Minimization (ERM) principle exploited by the ANN. Based on the SRM principle, the SVM possesses the advantage to control the model's complexity and generalization ability [58][60]. Thus the SVM are the very methods to deal with problems with limited samples and large dimensions better than the conventional machine-learning methods like the ANN. To avoid the main drawback of standard SVM, which is high computational complexity, Least Squares SVM (LS-SVM) was introduced by Suykens [61].

The LS-SVM is algorithmically more effective, because the solution uses equality constraints instead of inequality constraints and a least squares error term in order to obtain a linear set of equations in the dual space. But the traditional LSSVM is not suitable to model time-varying systems for its offline learning principle. The Online LS-SVM (OLS-SVM)[62] has showed encouraging promise for the ability of online adjusts the model parameters of time-varying systems. In [63] SVD based method for fetal ECG extraction has been proposed. Here this method performs singular value decomposition and based on the singular value ratio spectrum analysis FECG extraction has been done. SV decomposed modes of the appropriately configured data matrices identifies MECG components, which were eliminated to obtain FECG, through selective separation of the SV decomposed components. This method does not require any reference signal therefore it is computationally well efficient and robust.

Independent component analysis, also known as blind source separation,[64] is an interesting tool for the extraction of the antepartum fetal electrocardiogram from multi lead cutaneous potential recordings. This is nonparametric and is not based on pattern averaging, which could hamper the detection and analysis of atypical fetal heartbeats. Data-driven decomposition methods or spatial filtering techniques such as singular value decomposition (SVD) [65]-[68], Blind and semi blind source separation [69], construct basis functions from the data by maximizing some statistical measure of signal separation spatial filtering has an advantage over 
conventional adaptive filters because they can also extract the maternal and fetal complexes with temporal overlap.

Different types of blind and semi-blind source separation methods have been used for FECG signal separation normally these methods are based on the assumption of independent subspaces for the maternal and fetal signals, or the existence of some temporal structure for the fetal signals [70]-[72] . Blind Source separation methods can also be combined with wavelet decomposition methods [73],[74] for denoising and extracting FECG from composite abdominal ECG signal. These methods have an implicit assumption that both the signals and noises are mixed in a linear stationary manner which was considered as a main drawback, because the stationary mixing is often not a good approximation.

In [75] MICA based approach extraction method has presented which was more appropriate than ICA in fetal ECG extraction. The architecture of the proposed method includes preprocessing block, MECG estimation and post processing block. Here the preprocessing aimed to remove the baseline wander, EMG noise, power line interference by using FIR band pass filter and notch filter. The filter described in [76],[77] generated a synthetic MECG which tackled over the problem of MECG estimation.Estimated maternal signals were subtracted from AECG, however this approach fails when fetal ECG is weaker than the residual noise.

In [78] a fast and simple algorithm was developed based on ICA but computationally demanding calculations were substituted for making it simplex in FECG extraction. This approach consists of two steps. Initially dimensionality reduction is carried out followed by light post processing stage used to enhance the FECG signal. This algorithm is effective for the design of battery powered devices. In [79], a method was employed with the combination of singular value decomposition (SVD) and ICA for FECG signal separation from AECG. This method uses SVD of the spectrogram followed by an iterative application of ICA. In [79][82], ICA based BSS methods were used for extracting FECG, which showed that reconstruction of FECG could be possible by means of higher - order statistical tools.ICA based method for FECG extraction and MECG cancellation proposed in [83], worked efficiently even in $\mathrm{SNR}=-200 \mathrm{db}$ using simulated data without quantification noise, similarly the performance was decreased in the existence of quantification.

A method presented in [84] based on non-stationary ICA and wavelet de-noising. Due to low amplitude and poor signal to noise ratio (SNR) of the fetal ECG recorded at the abdominal region of a pregnant woman, the proposed algorithm removed the maternal ECG, reduced motion artifact and enhanced fetal ECG signal. Here due to the non-stationary nature of the fetal ECG signal, non-stationary ICA method was used to eliminate maternal complex then wavelet transform was used to remove baseline wander.In [85] a semi blind source separation algorithm has been developed, which requires prior information about the auto correlation function of the primary sources to extract the fetal ECG signal. They just assumed that the primary sources have a temporal structure and different autocorrelation functions, but not they are (primary sources) statistically independent. If there was any FHR variability, prior estimation of auto correlation function may not be appropriate for FHR analysis.

\subsection{Other Methods}

In [86] matching pursuits (MP) method has been proposed which extracted the low frequency periodic components of the complicated FHR patterns and also examined the long term modulation characteristics of the heart rate in relation to the oxygen saturation of fetal arterial blood. MP method efficiently detects abrupt perturbations and multiple periodicities in the FHR pattern.

A two stage successive cancellation algorithm has designed in [87] that separated fetal and maternal components to estimate the FHR in two stages each stage performs based on a template matching technique. The template of the stronger source that is MECG was cancelled from the composite ECG signal prior to the initialization of the weaker sources template in the second stage. Before the classification of weaker signals, classified events of the stronger source were removed, and then intervals between the detected adjacent fetal peaks were measured.

Based on digital filtering, adaptive thresholding, statistical properties in the time domain, and differencing of local maxima and minima an algorithm has been developed in [88] to determine fetal heart rate in real-time and for long-term monitoring with single lead configuration.In [89] polynomial networks technique for extraction was presented. In order to simplify the problem, they have assumed low noise electronic amplifiers with high common mode rejection ratio and classical low pass filtering techniques to eliminate the $50 \mathrm{~Hz}$ interference and to reduce EMG noise. Plynomial networks provide nonlinear mapping between maternal ECG and the component of maternal ECG in the abdominal signal. By aligning these two signals fetal ECG was extracted by subtracting the maternal ECG component from abdominal signal. Extraction was accomplished well in both non-overlapping and overlapping of FECG and MECG signals using this proposed technique.

In[90] extended version of linear prediction method has been used to remove maternal ECG from noninvasive fetal ECG recording. A new technique (SLP)was introduced and compared with the former technique which was Linear Prediction method (LP) by calculating the least mean square error (rms error) between the 
resulting FECG signals and the actual FECG signal, while the amplitude of the FECG signal is kept constant. A new non-blind method was proposed in [91] for FECG detection from multi channel abdominal recordings. This proposed sequential analysis method detected the FECG by estimating and removing the interference signals step-by-step, using a priori information about the interference signal and the signal of interest.

A three-stage methodology for the extraction of maternal and fetal heart rate using abdominal ECG leads, was presented in [92] Maternal R-peaks and fiducial points (maternal QRS onset and offset) were detected, using multiscale principal components analysis (MSPCA) and the Smoothed Nonlinear Energy Operator (SNEO) in the first stage,.Maternal fiducial points were used to eliminate the maternal QRS complexes from the abdominal ECG recordings. Again the same methods were employed to detect the fetal heart beats that do not overlap with the maternal QRSs (eliminated from the first stage). Finally histogram based technique was used for FHR detection.

In [93], multivariate singular spectrum analysis (MSSA) method was proposed for extracting and separating the mother heart signal, and fetal heart signal and the noise components from the combined ECGs.This algorithm composed of several complementary stages. First, the maternal ECG was extracted from a noisy composite signal using multivariate SSA by having thoracic signal as reference.Using MSSA, dynamic structure between the FECG and MECG signals were captured as a hidden complement information for the extraction of the FECG signal since the SSA technique consists of the elements of multivariate statistics, multivariate geometry and dynamical systemsA novel three-stage methodology was proposed in[94]. At first, using band-pass filtering and phase space analysis maternal R-peaks and fiducial points (maternal QRS onset and offset) were identified.In the second stage, bandpass filtering and multivariate Denoising were applied to enhance the fetalQRS complexes. The phase space characteristics were employed to identify fetal heart beats not overlapping with the maternal QRSsThe extraction of the fHR was accomplished in the third stage, using a histogram-based technique in order to identify the location of the fetal heart beats that overlap with the maternal QRSs.

A new method was proposed in [95] and evaluated a novel and flexible methodology for the analysis of FHR during labor. It is based on analyzing complexity of FHR data from a collection of multiscale and adaptive near-continuous piecewise linear approximations, obtained using a fast network flow algorithm. The measure of complexity could be used for detecting fetal per partum acidosis. In [96] automated methodology was proposed for the extraction of fetal heart rate using complex wavelets, time-frequency $(\mathrm{t}-\mathrm{f})$ analysis, and matching theory. It was a three-stage methodology in which maternal R-peaks and fiducial points (QRS onset and offset) were detected at the first stage using time-frequency $(\mathrm{t}-\mathrm{f})$ analysis and medical knowledge, to eliminate the maternal QRS from the input signals. Then the positions of the candidate fetal R-peaks were located using complex wavelets and matching theory techniques. Finally the fetal R-peaks, which overlap with the maternal QRS complexes (eliminated in the first stage) were found using two approaches a heuristic algorithm technique and a histogram based technique. Fetal heart rate was calculated by counting the detected $\mathrm{R}$ peaks.

In[97] a simulation system was presented to compare two adaptive filters based on recursive least square (RLS) and normalized least mean square (NLMS), in their use for fetal heart rate (FHR) monitoring. The reference(thoracic ECG) and primary signals(AECG) were fed simultaneously to the inputs of the RLS and NLMS adaptive filters to extract the fetal signal.In [98] a multi-step procedure was used to estimate the fECG signal single-lead abdominal signal.Initially maternal $\mathrm{R}$ peak locations were detected followed which each $\mathrm{R}-$ $\mathrm{R}$ interval in the abdominal signal was resampled to have the same number of samples by changing its corresponding sampling frequency. Comb filter was applied with the harmonics of the maternal electrocardiogram (mECG) over the resampled signal.Inorder to recover its original sampling frequency, eachR-R interval in the filtered signal was resampled again so that $\mathrm{mECG}$ signal was obtained,and then subtracted from the abdominal signal.A lightweight algorithm, was used in [99] to extract fetal ECG with a preknowledge about its skewness. By using skewness, cost function was defined by which weight vector updated and through this fetal ECG was extracted. Foetal electrocardiogram (FECG) extraction approach based on the cyclostationary properties of the signal of interest was carried out in [100].

\subsection{ICA For Twin FECG Extraction}

The algorithm proposed in [101] detects the fetal QRS complexes and converts the QRS onset series into a binary signal that is then recursively scanned to separate the contributions from the two fetuses. In [102],ICA based method was proposed for the separation of fetal ECG signal from magneto cardio-graphic signals in twin pregnancy. ICA used higher order statistics to decompose the input signal that is AECG, into statistical independent components. In[103] fastICA method was employed to extract twin fetal EMG from 55channel recording. 


\section{Conclusion}

The fetal electrocardiogram (FECG) was first demonstrated 114 years ago, initial progress of analysis in this area was limited. As improved amplifiers and filters became available, the detection of the waveform became easier, but observation of waveform morphology was complex due to the existing background noise after filtering the corrupted signal. The signal-to-noise ratio of the original FECG was increased considerably by efficient signal processing and computer techniques though the signals obtained non-invasively. In this survey paper various techniques for FECG signal extraction from the composite AECG signal were discussed, that gives up the various types of FECG signal analysis and extraction techniques so that accurate efficient methods can be applied during any clinical diagnosis, biomedical research.

Congenital Heart Defects. March of Dimes. 2005. [Online]. Available: http://www.marchofdimes.com/professionals/14332_1212.asp

[2]. Minino, AM.; Heron, MP.; Murphy, SL.; Kochanek, KD. Deaths: Final data for 2004. National Vital Statistics Reports. August. 2007

[3]. Congenital Heart Defects in Children Fact Sheet. American Heart Association; 2008. [Online].Available: http://www.americanheart.org/children

[4]. Pajkrt, E.; Weisz, B.; Firth, HV.; Chitty, LS. Fetal cardiac anomalies and genetic syndromes; Prenat Diagn. Dec. 2004 p. 1104 1115.[Online]. Available: http://dx.doi.org/10.1002/pd.1067.

[5]. Zuckerwar A, Pretlow R, Stoughton J, Baker D. Development of a piezopolymer pressure sensor for a portable fetal heart rate monitor. IEEE Trans Biomed Eng. 1993; 40(no. 9):963-969. [PubMed:8288288]

[6]. Peters M, Crowe J, Piéri JF, Quartero H, Hayes-Gill B, James D, Stinstra J, Shakespeare S.Monitoring the fetal heart noninvasively: a review of methods. J Perinat Med. 2001; 29:408-416.[PubMed: 11723842]

[7]. K. V. K. Ananthanag and J. S. Sahambi, "Investigation of Blind Source Separation Methods for Extraction of Fetal ECG," CCECE 2003-CCGEI 2003, pp.2021-2024, May.2003

[8]. Naheed, ZJ, Strasburger JF, Deal BJ, Benson, DW, Gidding SS (1996) Fetal tachycardia: mechanisms and predictors of hydrops fetalis. J Am Coll Cardiol 27: 1736-1740.

[9]. Simpson, JM, Sharland GK (1998) Fetal tachycardias: management and outcome of 127 consecutive cases. Heart 79: 576-581.

[10]. Jaeggi ET, Carvalho JS, De Groot E, Api O, Clur SA, et al. (2011) Comparison of transplacental treatment of fetal supraventricular tachyarrhythmias with digoxin, flecainide, and sotalol: results of a nonrandomized multicenter study. Circulation 124: 1747-1754.

[11]. Jaeggi ET, Hamilton RM, Silverman ED, Zamora SA, Hornberger LK (2002) Outcome of children with fetal, neonatal or childhood diagnosis of isolated congenital atrioventricular block. A single institution's experience of 30 years. J Am Coll Cardiol 39: 130-137.

[12]. Jaeggi ET, Hornberger LK, Smallhorn JF, Fouron JC (2005) Prenatal diagnosis of complete atrioventricular block associated with structural heart disease: combined experience of two tertiary care centers and review of the literature. Ultrasound Obstet Gynecol 26: 16.

[13]. Breuer JM, Gooskens RH, Kapusta L, Stoutenbeek P, Visser GH, et al. (2007) Neurological outcome in isolated congenital heart block and hydrops fetalis. Fetal Diagn Ther 22: 457-461.

[14]. Oudijk MA, Gooskens RH, Stoutenbeek P, De Vries LS, Visser GH, et al. (2004) Neurological outcome of children who were treated for fetal tachycardia complicated by hydrops. Ultrasound Obstetr Gynecol 24: 154-158.

[15]. Widrow B, Glover J, McCool J, Kaunitz J, Williams C, Hearn H, Zeidler J, Dong E, Goodlin R.Adaptive noise cancelling: principles and applications. Proc IEEE. 1975; 63(no. 12):1692-1716.

[16]. Outram NJ, Ifeachor EC, Eetvelt PWJV, Curnow JSH. Techniques for optimal enhancement and feature extraction of fetal electrocardiogram. IEE Proc-Sci Meas Technol. November; 1995 142(no. 6):482-489.

[17]. G. Mihaela Ungureanu, Ana Maria Ilincai, Werner Wolf, Ilinca Gussi, Dragos D. Taralunga, Rodica Strungaru Improved Fetal ECG Extraction by Applying Adaptive Filtering,International symposium on ADVANCED TOPICS IN ELECTRICAL ENGINEERING,May 12-14,2011

[18]. Prasanth K., Baby Paul, Arun A. Balakrishnan, Fetal ECG Extraction Using Adaptive Filters, International Journal of Advanced Research in Electrical, Electronics and Instrumentation Engineering Vol. 2, Issue 4, April 2013

[19]. Farvet AG. Computer Matched Filter Location of Fetal R-Waves. Medical \& Biological Engineering. September; 1968 6(no. 5):467-475

[20]. Park Y, Lee K, Youn D, Kim N, Kim W, Park S. On detecting the presence of fetal R-wave using the moving averaged magnitude difference algorithm. IEEE Trans Biomed Eng. August; 1992 39(no. 8):868-871. [PubMed: 1506000]

[21]. Martens, SMM.; Rabotti, C.; Mischi, M.; Sluijter, RJ. A robust fetal ECG detection method for abdominal recordings; Physiol Meas. Apr. 2007 p. 373-388.[Online]. Available:http://dx.doi.org/10.1088/0967-3334/28/4/004

[22]. Shao M, Barner K, Goodman M. An interference cancellation algorithm for noninvasive extraction of transabdominal fetal electroencephalogram (TaFEEG). IEEE Trans Biomed Eng. 2004; 51(no.3):471-483. [PubMed: 15000378]

[23]. Sameni, R. PhD dissertation. Sharif University of Technology - Institute National Polytechnique de Grenoble; July. 2008 Extraction of Fetal Cardiac Signals from an Array of Maternal Abdominal Recordings.[Online].Available: http://www.sameni.info/Publications/Thesis/PhDThesis.pdf

[24]. Sameni R, Shamsollahi MB, Jutten C, Clifford GD. A Nonlinear Bayesian Filtering Framework for ECG Denoising. IEEE Trans Biomed Eng. Dec; 2007 54(no. 12):2172-2185. [PubMed:18075033]

[25]. Sameni R, Shamsollahi MB, Jutten C. Model-based Bayesian filtering of cardiac contaminants from biomedical recordings. Physiological Measurement. May; 2008 29(no. 5):595-613.[PubMed: 18460766]

[26]. Mohammad Niknazar, Bertrand Rivet, and Christian Jutten Fetal ECG Extraction by Extended State Kalman Filtering Based on Single-Channel Recordings IEEE TRANSACTIONS ON BIOMEDICAL ENGINEERING, VOL. 60, NO. 5, MAY 20131345

[27]. R. Sameni,M. B. Shamsollahi, C. Jutten, and G. D. Clifford, “A nonlinear Bayesian filtering framework for ECG denoising," IEEE Trans. Biomed. Eng., vol. 54, no. 12, pp. 2172-2185, Dec. 2007.

[28]. R.Swarnalatha ,D.V.Prasad,A Novel Technique for Extraction of FECG using Multistage Adaptive Filtering,Journal of Applied Sciences 10(4):319-324,2010 ISSN 1812-5654

[29]. Widrow B., Stearns S. (1985) Adaptive signal processing. Prentice-Hall, Upper Saddle River. 
[30]. Datian, Y., Xuemei, O. (1996) Application of wavelet analysis in detection of fetal ECG. Engineering in Medicine and Biology Society. Bridging Disciplines for Biomedicine.Proceedings of the 18th Annual International Conference of the IEEE.Amsterdam. Netherlands; 31 October-3 November 1996. vol. 3. p. 1043-1044.

[31]. Echeverria, J. C., Ramirez, N., Pimentel, A.B., et al. (1996) Fetal QRS extraction based on wavelet analysis and pattern matching.Engineering in medicine and biology society.Bridging disciplines for biomedicine.Proceedings of the 18th Annual International Conference of the IEEE. Amsterdam.Netherlands; 31 October-3 November 1996. vol. 4. p. $1656-1657$.

[32]. Mallat S., Hwang W. L. (1992) Singularity detection and processing with wavelets.IEEE Trans Inf Theory 38:617-643

[33]. Papadimitriou, S. , Gatzounas, D. ,Papadopoulos, V., et al. (1996) Fetal heart rate signal denoising by processing the wavelet transform modulus maxima.Engineering in Medicine and Biology Society, Bridging Disciplines for Biomedicine.Proceedings of the 18th Annual International Conference of theIEEE. Amsterdam. Netherlands; 31 October-3 November 1996. vol. 3. p.1029-1030.

[34]. Mochimaru F., Fujimoto Y. (2002) Detecting the fetal electrocardiogram by wavelet theory-based methods. Prog Biomed Res 7:185-193.

[35]. Karvounis, E. C., Papaloukas, C., Fotiadis,D. I., et al. (2004) Fetal heart rate extraction from composite maternal ECG using complex continuous wavelet transforms. Comput Cardiol 737-740. Chicago, IL, USA.

[36]. Song, Y., Xie, W., Chen, J. F., et al. (2006) Passive acoustic maternal abdominal fetal heart rate monitoring using wavelet transform. Comput Cardiol 581-584, Valencia, Spain.

[37]. Karvounis. E. C., Tsipouras, M. G., Fotiadis, D. I., et al. (2006) A method for fetal heart rate extraction based on time-frequency analysis. 19th IEEE Symposium on Computer-Based Medical Systems (CBMS'06). Salt Lake City, UT, USA; $22-23$ June 2006. p.347-347.

[38]. B. Azzerboni, F. Foresta, N. Mammone and F. C. Morabito, “A New Approach BasedOn Wavelet-ICA Algorithm for Fetal Electrocardiogram Extraction,” presented at the European Symp. Artificial Neural Networks, Bruges, Apr. 2005.

[39]. S Almagro, MM. Elena, MJ. Bastiaans and JM. Quero, “A New Mother Wavelet for Fetal electrocardiography, to Achieve Optimal De-noising and Compressing results," in computers in cardiology, 2006. pp. 157-160.

[40]. S. Kadambe, R. Murray and F. Boudreaux-Bartels, "Wavelet Transform-Based QRS Complex Detector," IEEE Trans. Biomed. Eng., vol. 46, no. 7, pp. 838-847, Jul.1999

[41]. K.D. Desai,Manoj S. Sankhe A Real-Time Fetal ECG Feature Extraction Using Multiscale Discrete Wavelet Transform 2012 5th International Conference on BioMedical Engineering and Informatics (BMEI 2012)

[42]. Liszka-Hackzell, J. (1994) Categorization of fetal heart rate patterns using neural networks.Comput Cardiol 97-100. Bethesda, Maryland, USA.

[43]. Marques de Sa, J. P., Reis, L. P., Lau, J. N.et al. (1994) Estimation and classification of fetal heart rate baselines using artificial neuralnetworks. Comput Cardiol 541-544.Bethesda, Maryland, USA.

[44]. Magenes. G., Signorini, M. G., Arduini, D.(1999) Detection of normal and pathological fetal states by means of neural and fuzzy classifiers applied to CTG parameters. Engineering in Medicine and Biology. 21st Annual Conference and the 1999 Annual Fall Meeting of the Biomedical Engineering Soc. BMES/EMBS Conference. Atlanta, GA, USA; 13-16 October 1999. vol. 2. p.936-936.

[45]. Selvan, S., Srinivasan, R. (2000) A novel adaptive filtering technique for the processing of abdominal fetal electrocardiogram using neural network. Adaptive systems for signal processing, communications and control symposium 2000. Louise, Alta,Canada; p. 289-292.

[46]. Camps, G., Martinez, M., Soria, E. (2001) Fetal ECG extraction using an FIR neural network. Comput Cardiol 249-252. Rotterdam, Netherlands.

[47]. Widrow B., Glover J. R. Jr, McCool J. M.et al. (1975) Adaptive noise canceling: principlesand applications. Proc IEEE 63:16921716

[48]. Reaz, M. B. I., Wei, L. S. (2004) Adaptive linear neural network filter for fetal ECGextraction. Proceedings of international conference on intelligent sensing and information processing. Chennai. India; 4-7January 2004, p. 321-324.

[49]. Warrick, P., Hamilton, E., Macieszczak, M.(2005) Neural network based detection of fetal heart rate patterns. Proceedings of International Joint Conference on NeuralNetworks. Montreal. Canada; 31 July-4 August 2005. vol. 4. p. 2400-2405.

[50]. L. Xia, J. Meng, R. Xu, B. Yan, Y. Guo, Modeling of 3-D vertical interconnect using support vector machine regression, IEEE Microw. Wireless Compon. Lett., vol. 16, no. 1, Dec. 20062,639-641

[51]. M. S. Amin, Md. Mamun, F. H. Hashim and H. Husain Separation of fetal electrocardiography (ECG) from composite ECG using adaptive linear neural network for fetal monitoring International Journal of the Physical Sciences Vol. 6(24), pp. 5871-5876, 16 October, 2011 Available online at http://www.academicjournals.org/IJPS

[52]. Maryam Nasiri Karim Faez Extracting Fetal Electrocardiogram Signal UsingANFIS Trained by Genetic Algorithm International Conference on Biomedical Engineering (ICoBE),27-28 February 2012

[53]. M. S. Amin, Md. Mamun, F. H. Hashim and H. Husain Separation of fetal electrocardiography (ECG) from composite ECG using adaptive linear neural network for fetal monitoring International Journal of the Physical Sciences Vol. 6(24), pp. 5871-5876, 16 October, 2011DOI: 10.5897/IJPS11.1432 Available online at http://www.academicjournals.org/IJPS

[54]. Maryam Nasiri , Karim Faez, Ali Motie Nasrabadi A New Method for Extraction of Fetal Electrocardiogram Signal Based on Adaptive Nero-Fuzzy Inference System 2011 IEEE International Conference on Signal and Image Processing Applications (ICSIPA2011)

[55]. Maryam Nasiri , Karim Faez, Ali Motie Nasrabadi A New Method for Extraction of Fetal Electrocardiogram Signal Based on Adaptive Nero-Fuzzy Inference System 2011 IEEE International Conference on Signal and Image Processing Applications (ICSIPA2011)

[56]. K. Assaleh, "Extraction of Fetal Electrocardiogram Using Adaptive Neuro-Fuzzy Inference System," IEEE Trans. Biomed. Eng., vol. 54, no. 1, pp. 59-68, Jan. 2007.

[57]. Kezi Selva Vijila, C., Kanagasabapathy, P.,Johnson, S. (2005) Adaptive neuro fuzzy inference system for extraction of FECG. Proceedings of IEEE India Annual Conference (INDICON 2005). Chennai, India;11-13 December 2005. p. $224-227$.

[58]. L. Xia, J. Meng, R. Xu, B. Yan, Y. Guo, Modeling of 3-D vertical interconnect using support vector machine regression, IEEE Microw. Wireless Compon. Lett., vol. 16, no. 1, Dec. 20062,639-641

[59]. V. N. Vapnik, The Nature of Statistical Learning Theory, New York: Springer-Verlag, 1995, Ch.6,181-183

[60]. V. N. Vapnik, An overview of statistical learning theory, IEEE Trans. Neural Networks, vol. 10,no. 5, Sep. 1999, 988-999

[61]. M. S. Lee, S. S. Keerthi, C. J. Ong, et al. An efficient method for computing leave-one-out error in support vector machines with Gaussian kernels. IEEE Trans. Neural Networks, 15(3), 2004,750-757

[62]. Liang Han, Xiujuan Pu, Na Zhou, Bowei Zhang, Wenhao Jiang Extraction of Fetal Electrocardiogram Using Online Least Squares Support Vector Machines, Journal of Information \& Computational Science 8: 11 (2011) 2045-2057 Available at http://www.joics.com 
[63]. Kanjilal P. P., Palit S., Saha G. (1997) Fetal ECG extraction from single-channel maternal ECG using singular value decomposition. IEEE Trans Biomed Eng 44:51-59

[64]. Lieven De Lathauwer, Bart De Moor, and Joos Vandewall Fetal Electrocardiogram Extraction by Blind Source Subspace Separation IEEE TRANSACTIONS ON BIOMEDICAL ENGINEERING, VOL. 47, NO. 5, MAY 2000 van Oosterom A. Spatial filtering of the fetal electrocardiogram. J Perinat Med. 1986; 14(no. 6):411-419. [PubMed: 3820041]

[65]. Damen AA, Van Der Kam J. The use of the singular value decomposition in electrocardiography.

[66]. Med Biol Eng Comput. July; 1982 20(no. 4):473-82. [PubMed: 7132453]

[67]. Vanderschoot J, Callaerts D, Sansen W, Vandewalle J, Vantrappen G, Janssens J. Two methods for optimal MECG elimination and FECG detection from skin electrode signals. IEEE Trans Biomed Eng. 1987; BME-34:233-243. [PubMed: 3570313]

[68]. Callaerts, D. PhD dissertation. K.U.Leuven - E.E. Dept.; Dec. 1989 Signal separation methods based on singular value decomposition and their application to the real-time extraction of the fetal electrocardiogram from cutaneous recordings.

[69]. K. V. K. Ananthanag and J. S. Sahambi, "Investigation of Blind Source Separation Methods for Extraction of Fetal ECG," CCECE 2003-CCGEI 2003, pp.2021-2024, May.2003

[70]. Kanjilal PP, Palit S, Saha G. Fetal ECG extraction from single-channel maternal ECG using singular value decomposition. IEEE Trans Biomed Eng. Jan.1997 44:51-59. [PubMed: 9214783]

[71]. Zarzoso V, Nandi AK, Bacharakis E. Maternal and fetal ECG separation using blind source separation methods. IMA J Math Appl Med Biol. 1997; 14:207-225. [PubMed: 9306675]

[72]. Zhang ZL, Yi Z. Extraction of temporally correlated sources with its application to noninvasive fetal electrocardiogram extraction. Neurocomputing. 2006; 69(no. 7-9):894-899.

[73]. Li Y, Yi Z. An algorithm for extracting fetal electrocardiogram. Neurocomput. 2008; 71(no. 7-9): 1538-1542.Sameni and Clifford Page 21

[74]. Barros, AK.; Cichocki, A. Extraction of specific signals with temporal structure; Neural Comput.Sep. 2001 p. 1995-2003.[Online]. Available: http://dx.doi.org/10.1162/089976601750399272

[75]. J. L. Camargo-Olivares, R. Martín-Clemente, Member, IEEE, S. Hornillo-Mellado, M. M. Elena, and I. RománThe Maternal Abdominal ECG as Input to MICA in the Fetal ECG Extraction Problem IEEE SIGNAL PROCESSING LETTERS, VOL. 18, NO. 3, MARCH 2011

[76]. J. F. Cardoso, "Multidimensional independent component analysis," in Proc. ICASSP'98, Seattle, 1998, pp. 1941-1944.

[77]. R. Sameni, M. Shamsollahi, C. Jutten, and G. Clifford, "A nonlinear bayesian filtering framework for ECG denoising," IEEE Trans.Biomed. Eng., vol. 54, no. 12, pp. 2172-2185, Dec. 2007.

[78]. Ruben Mart'1n-Clemente, Member, IEEE, Jose Luis Camargo-Olivares, Susana Hornillo-Mellado, Mar Elena,and Isabel Rom'an Fast Technique for Noninvasive Fetal ECG Extraction, IEEE TRANSACTIONS ON BIOMEDICAL ENGINEERING, VOL. 58, NO. 2, FEBRUARY 2011

[79]. Ping, G., Ee-Chien, C., Wyse, L. (2003) Blind separation of fetal ECG from single mixture using SVD and ICA. 4th International Conference on Information, Communications \& Signal Processing and $4^{\text {th }}$ Pacific-Rim Conference on Multimedia (ICICS-PCM 2003). Singapore; 15-18 December 2003. vol. 3. p. 1418-1422.

[80]. Vigneron,V., Paraschiv-Ionescu, A.,Azancot, A., et al. (2003) Fetal electrocardiogram extraction based on non-stationary ICA and wavelet denoising, proceedings. 7th International Symposium on Signal Processing and Its Applications. Paris, France. 1-4 July 2003. vol. 2. p. 69-72.

[81]. de Araujo, D., Barros, A. K., Baffa, O., et al.(2003) Fetal magnetocardiographic source separation using the poles of the autocorrelation function. Proceedings of the 4th international symposium on independent component analysis and blind signal separation,Nara, Japan, 1-4 April 2003, pp. 833-836.

[82]. Xinling Wen, Zhengzhou Institute of Aeronautical Industry Management, Henan, Zhengzhou Performance Comparison Research of the FECG Signal Separation Based on the BSS Algorithm Research Journal of Applied Sciences, Engineering and Technology 4(16): 2800-2804, 2012 ISSN: 2040-7467

[83]. Najafabadi, F. S., Zahedi, E., Ali, M. A. M.(2005) A novel model for abdominal electrocardiography of a pregnant woman. Sens ors and the international conference on new techniques in pharmaceutical and biomedical research 64-68. Kuala Lumpur, Malaysia.

[84]. G. Mihaela Ungureanu, Ana Maria Ilincai, Werner Wolf, Ilinca Gussi, Dragos D. Taralunga, Rodica Strungaru Improved Fetal ECG Extraction by Applying Adaptive Filtering,International symposium on ADVANCED TOPICS IN ELECTRICAL ENGINEERING,May 12-14,2011

[85]. Barros A. K., Cichocki A. (2001) Extraction of specific signals with temporal structure. Neural Comput 13:1995-2003 MIT Press.

[86]. Vasios, G., Prentza, A., Hintipas, E., et al. (2003) Investigating the effect of oxygen saturation on very low frequency periodic components of the fetal heart rate during labor using matching pursuits. Proceedings of the 25th Annual International Conference of the IEEE in Engineering in Medicine and Biology Society. Cancun. Mexico;17-21 September 2003. vol. 3. pp. 2566-2569.

[87]. Laim K. C., Shynk J. J. (2002) A successive cancellation algorithm for fetal heart-rate estimation using an intrauterine ECG signal.IEEE Trans Biomed Eng 49:943-954.

[88]. M. I. Ibrahimy, F. Ahmed, M. A. Mohd and E. Zahedi, "Real-time Signal Processing for Fetal Heart Rate Monitoring," IEEE Trans. Biomed. Eng., vol. 50, no. 2, pp. 258-262, Feb. 2003.

[89]. K. Assaleh and H. Al-Nashash, "A novel technique for the extraction of fetal ECG using polynomial networks," IEEE Trans. Biomed. Eng., vol. 52, no. 6, pp. 1148-1152, Jun. 2005.

[90]. R. Vullings, C. Peters, M. Mischi, G. Oie and J Bergmans, "Maternal ECG Removal from Non-Invasive Fetal ECG Recordings," IEEE, 2nd EMBS annual international conference, New York City, Aug. 2006.

[91]. Suzanna M M Martens, Chiara Rabotti, Massimo Mischi and Rob J SluijterA robust fetal ECG detection method for abdominal recordings IOP PUBLISHING PHYSIOLOGICAL MEASUREMENT Physiol. Meas. 28 (2007) 373-388 doi:10.1088/0967$3334 / 28 / 4 / 004$

[92]. Evaggelos C. Karvounis, Student Member, IEEE and Dimitrios I. Fotiadis, Senior Member, IEEE Maternal and fetal heart rate extraction from abdominal recordings using multi-scale principal components analysis Proceedings of the 29th Annual International Conference of the IEEE EMBS August 23-26, 2007

[93]. Mansoureh Ghodsi, Hossein Hassani and Saeid Sanei Extracting fetal heart signal from noisy maternal ECG by multivariate singular spectrum analysis Statistics and Its Interface Volume 3 (2010) 399-411

[94]. Evaggelos C. Karvounis, Member, IEEE, Markos G. Tsipouras, Member, IEEE, and Dimitrios I. Fotiadis, Senior Member, IEEE Detection of Fetal Heart Rate Through 3-D Phase Space Analysis From Multivariate Abdominal Recordings IEEE TRANSACTIONS ON BIOMEDICAL ENGINEERING, VOL. 56, NO. 5, MAY 2009

[95]. H. Helgason*, P. Abry, P. Gonc,alv`es, Cl. Gharib, P. Gaucherand, and M. D Adaptive Multiscale Complexity Analysis of Fetal Heart Rate IEEE TRANSACTIONS ON BIOMEDICAL ENGINEERING, VOL. 58, NO. 8, AUGUST 2011 
[96]. Evaggelos C. Karvounis, Student Member, IEEE, Markos G. Tsipouras, Student Member, IEEE,Dimitrios I. Fotiadis, Senior Member, IEEE, and Katerina K. Naka An Automated Methodology for Fetal Heart Rate Extraction From the Abdominal Electrocardiogram IEEE TRANSACTIONS ON INFORMATION TECHNOLOGY IN BIOMEDICINE, VOL. 11, NO. 6, NOVEMBER 2007

[97]. M. M. Sheikh Algunaidi, M. A. Mohd Ali and Md. Fokhrul Islam, Comparative analysis of fetal electrocardiogram (ECG) extraction techniques using system simulation International Journal of the Physical Sciences Vol. 6(21), pp. 4952-4959, 30 September, 2011 Available online at http://www.academicjournals.org/IJPS

[98]. Zheng Wei, Liu Hongxing, He Aijun, Ning Xinbao, Cheng Jianchun Single-lead fetal electrocardiogram estimation by means of combining R-peak detection, resampling and comb filter, Medical Engineering \& Physics 32 (2010) 708-719

[99]. Fahimeh Jafari, Mohammad A Tinati, Behzad Mozaffar A New Fetal ECG Extraction Method Using its Skewness Value Which lies in Specific Range Proceedings of ICEE 2010, May 11-13, 2010

[100]. Michel Haritopoulos,Cécile Capdessus, Asoke K. Nandi Foetal PQRST Extraction from ECG Recordings using CyclostationarityBased Source Separation Method 32nd Annual International Conference of the IEEE EMBS Buenos Aires, Argentina, August 31 September 4, 201

[101]. A. Fanelli, Student Member, IEEE, M.G. Signorini, Member, IEEE, and T. Heldt, Senior Member, IEEE Extraction of Fetal Heart Rate from Maternal Surface ECG with Provisions for Multiple Pregnancies, 34th Annual International Conference of the IEEE EMBS San Diego, California USA, 28 August - 1 September, 2012

[102]. M. Burghoff, P. Van Leeuwen, "Separation of Fetal and Maternal Magnetocardiographic Signals in Twin Pregnancy Using Independent Component Analysis (ICA),', Neurology and Clinical neurophYSiology, vol. 39, pp. 1-4, 2004.

[103]. S. Comani, D. Mantini, G. Alleva, E. Gabriele, M. Liberati and G.L. Romani, "Simultaneous monitoring of separate fetal magnetocardiographic signals in twin pregnancy", Physiological Measurement, Vol. 26, pp. 193-201, 2005. 